\title{
BICROSSED PRODUCTS WITH THE TAFT ALGEBRA
}

\author{
A. L. AGORE AND L. NĂSTĂSESCU
}

\begin{abstract}
Aвstract. Let $G$ be a group which admits a generating set consisting of finite order elements. We prove that any Hopf algebra which factorizes through the Taft algebra and the group Hopf algebra $K[G]$ (equivalently, any bicrossed product between the aforementioned Hopf algebras) is isomorphic to a smash product between the same two Hopf algebras. The classification of these smash products is shown to be strongly linked to the problem of describing the group automorphisms of $G$. As an application, we completely describe by generators and relations and classify all bicrossed products between the Taft algebra and the group Hopf algebra $K\left[D_{2 n}\right]$, where $D_{2 n}$ denotes the dihedral group.
\end{abstract}

\section{INTRODUCTION}

The bicrossed product construction first emerged in group theory, under the name of Zappa-Szép product or knit product, as a natural generalization of the semi-direct product; see for instance [19, Section 2] where the terminology adopted in the sequel originates. More precisely, any bicrossed product of groups is constructed from a so-called matched pair of groups, i.e. a pair of groups $(G, H)$ which act on each other by means of two actions subject to some very natural compatibility conditions (see [19. Definition 2.1]). The two actions of a matched pair induce a group structure on the direct product $G \times H$ of the underlying sets, called the bicrossed product of $G$ and $H$. Furthermore, it was shown that the bicrossed product provides the answer to the factorization problem in the sense that any group which factorizes through two given groups is isomorphic to a bicrossed product between the same groups. Due to the interest generated by its connection to the factorization problem, the bicrossed product construction was considered in varied contexts (e.g. for (co)algebras [8], [9], Lie algebras [15], [16], Hopf algebras [17] etc.) and nowadays is being studied for many different purposes. For instance, in the case of associative algebras this construction is often referred to as twisted tensor product and it seems to provide the proper algebraic counterpart for the product of noncommutative spaces under the duality between the categories of commutative algebras and algebraic affine spaces (see [12], [18] for further details). Another strong motivation for studying bicrossed products stems from their link to the (quantum) Yang-Baxter equation; more precisely, there are several methods to produce solutions for the Yang-Baxter equation by means of matched pairs and the corresponding bicrossed products (see e.g. [6], [10]). Furthermore, the bicrossed product construction turns out to be general enough to recover as special cases many of the classical constructions in group theory (e.g. semi-direct products), associative algebras (e.g. skew group algebras, Ore extensions), Lie algebras (complex product structures [5]) or Hopf algebras (e.g. the Drinfel'd double, the generalized quantum double). However, even in the simplest cases the problem of describing and classifying all bicrossed products between two given objects (groups, associative algebras, Lie algebras etc.) turns out to be very difficult. Probably the

2010 Mathematics Subject Classification. 16T05, 16S40.

Key words and phrases. Bicrossed product, the factorization problem, classification of Hopf algebras, Taft algebra. A.L. Agore is Postdoctoral fellow of FWO-Vlaanderen. 
most conclusing argument comes from the group case where the description and classification of all bicrossed products between two finite cyclic groups is still an open question (see [4] for an up-to-date account on the subject). Nevertheless, this topic has been intensively studied in several papers including [1], [3], [7], [8], [9], [12], [18], to mention only a few, and it turned out to be an effective method of constructing new objects (groups, associative algebras, Hopf algebras etc.) out of given ones.

The present study continues the work of [3] where a strategy for classifying bicrossed products of Hopf algebras was proposed. This avenue of investigation based on the description of all morphisms between two bicrossed products was previously pursued in [1], [2], [7], [14] and led to promising results. The paper is structured as follows. Section 1 gives a brief review of some preparatory results used in the sequel while the main results are contained in Section 2, To start with, if $G$ is a group which admits a generating set consisting of finite order elements, then Theorem 2.1 proves that any bicrossed product between the Taft algebra and the group Hopf algebra $K[G]$ is in fact a smash product between the same two Hopf algebras. Although we are able to prove that the right action of any such bicrossed product is always trivial, the description of the left action depends essentially on the relations, if any, satisfied by the generators of $G$. Along the way, we describe by generators and relations all matched pairs between the Taft algebra and the group Hopf algebra $K\left[D_{l n}^{k}\right]$, where $D_{l n}^{k}$ is the semidirect product of cyclic groups defined in (11). More precisely, these are implemented by two roots

of unity $\beta \in U_{l}(K), \sigma \in U_{(n, k-1)}(K)$ and the corresponding smash products, denoted by $T_{l n m^{2}}^{\beta, \gamma}(q)$, are described by generators and relations in Theorem 2.2

The classification of the smash products between the Taft algebra and the group Hopf algebra $K[G]$ is shown to be strongly linked to the problem of describing the group automorphisms of $G$ (Theorem 2.3. Since the problem of describing the automorphisms of a given group is widely open and it leads to cumbersome computations even for the groups $D_{l n}^{k}$ (see [11]), in this paper we restrict to the classification of Hopf algebras which factorize through the Taft algebra and the group Hopf algebra $K\left[D_{2 n}\right]$. More precisely, Theorem 2.4 provides necessary and sufficient conditions for two bicrossed products between the Taft Hopf algebra and $K\left[D_{2 n}\right]$ to be isomorphic while Theorem 2.5 counts the number of types of these bicrossed products which is shown to depend on the arithmetics of $m$ and $n$.

\section{Preliminaries}

Throughout $K$ will be a field. Unless specified otherwise, all algebras, coalgebras, bialgebras, Hopf algebras, tensor products and homomorphisms are over $K$. For a coalgebra $(C, \Delta, \varepsilon)$ and $y \in C$, we use Sweedler's $\Sigma$-notation: $\Delta(y)=y_{(1)} \otimes y_{(2)},(I \otimes \Delta) \Delta(y)=y_{(1)} \otimes y_{(2)} \otimes y_{(3)}$, etc (summation understood). Let $A$ and $H$ be two Hopf algebras. $H$ is called a left (resp. right) $A$-module (co)algebra if $H$ is a (co)algebra in the monoidal category of left (resp. right) $A$-modules.

For a positive integer $n$ we denote by $U_{n}(K)$ the cyclic group of $n$-th roots of unity in $K$ and by $\left|U_{n}(K)\right|$ its order. Whenever $\left|U_{n}(K)\right|=n$, any generator of $U_{n}(K)$ is called a primitive $n$-th root of unity. $C_{n}$ stands for the cyclic group of order $n$ while $(m, n)$ denotes the greatest common divisor of $m, n \in \mathbb{N}^{*}$. Furthermore, given integers $k, l, n \in \mathbb{N}^{*}$ with $(k, n)=1$ we denote by $D_{l n}^{k}$ the semi-direct product of groups:

$$
C_{n} \rtimes_{k} C_{l}=<c, d \mid c^{l}=1, d^{n}=1, c d=d^{k} c>
$$

If $l=2$ and $k=n-1$ we recover the dihedral groups which will be denoted simply by $D_{2 n}$. Given a group $G, K[G]$ stands for the group Hopf algebra on $G$. If $H$ is a Hopf algebra then $\mathcal{G}(H)$ denotes the 
group-like elements of $H$. Furthermore, for any $g, h \in \mathcal{G}(H)$ we denote by $\mathcal{P}_{g, h}(H)$ the set of $(g, h)$ skew primitive elements of $H$, i.e. $y \in \mathcal{P}_{g, h}(H)$ if $\Delta(y)=y \otimes g+h \otimes y$.

Consider $m \in \mathbb{N}, m \geq 2$; since the Taft Hopf algebra $T_{m^{2}}(q)$ plays a central role in this paper, we will assume that the base field $K$ contains a primitive $m$-th root of unity $q$. Recall that the Taft Hopf algebra of order $m$ over $K$ is generated as an algebra by two elements $h$ and $x$ subject to the relations $h^{m}=1$, $x^{m}=0$ and $x h=q h x$. The coalgebra structure is such that $h$ is a group-like element and $x$ is a $(h, 1)$ skew primitive element. Furthermore, $\left\{h^{i} x^{j}\right\}_{0 \leq i, j \leq m-1}$ is a $K$-linear basis of the Taft algebra, the set of group-like elements is $\mathcal{G}\left(T_{m^{2}}(q)\right)=\left\{h^{i} \mid i \in 0, \cdots, m-1\right\}$ and the $\left(h^{j}, 1\right)$-skew primitive elements are given as follows for any $j=\{0,1, \cdots, m-1\}$ :

$$
\mathcal{P}_{h^{j}, 1}\left(T_{m^{2}}(q)\right)=\left\{\begin{aligned}
\alpha\left(h^{j}-1\right), & \text { if } \quad j \neq 1 \\
\beta(h-1)+\gamma x, & \text { if } \quad j=1
\end{aligned} \text {, for some } \alpha, \beta, \gamma \in K\right.
$$

A matched pair of Hopf algebras is a quadruple $(A, H, \triangleleft, \triangleright)$, where $A$ and $H$ are Hopf algebras, $\triangleleft$ : $H \otimes A \rightarrow H, \triangleright: H \otimes A \rightarrow A$ are linear maps such that $(A, \triangleright)$ is a left $H$-module coalgebra, $(H, \triangleleft)$ is a right $A$-module coalgebra and the following compatibilities hold for any $a, b \in A, y, z \in H$.

$$
\begin{aligned}
y \triangleright 1_{A} & =\varepsilon_{H}(h) 1_{A}, 1_{H} \triangleleft a=\varepsilon_{A}(a) 1_{H} \\
y \triangleright(a b) & =\left(y_{(1)} \triangleright a_{(1)}\right)\left(\left(y_{(2)} \triangleleft a_{(2)}\right) \triangleright b\right) \\
(y z) \triangleleft a & =\left(y \triangleleft\left(z_{(1)} \triangleright a_{(1)}\right)\right)\left(z_{(2)} \triangleleft a_{(2)}\right) \\
y_{(1)} \triangleleft a_{(1)} \otimes y_{(2)} \triangleright a_{(2)} & =y_{(2)} \triangleleft a_{(2)} \otimes y_{(1)} \triangleright a_{(1)}
\end{aligned}
$$

Any matched pair of Hopf algebras $(A, H, \triangleleft, \triangleright)$ gives rise to a new Hopf algebra on the tensor coalgebra $A \otimes H$ with the multiplication and antipode given for all $a, b \in A, y, z \in H$ as follows:

$$
\begin{aligned}
(a \bowtie y) \cdot(b \bowtie z) & =a\left(y_{(1)} \triangleright b_{(1)}\right) \bowtie\left(y_{(2)} \triangleleft b_{(2)}\right) z \\
S_{A \bowtie H}(a \bowtie y) & =S_{H}\left(y_{(2)}\right) \triangleright S_{A}\left(a_{(2)}\right) \bowtie S_{H}\left(y_{(1)}\right) \triangleleft S_{A}\left(a_{(1)}\right)
\end{aligned}
$$

where we denote $a \otimes y$ by $a \bowtie y$. This new Hopf algebra is called the bicrossed product associated to the matched pair of Hopf algebras $(A, H, \triangleleft, \triangleright)$ and it will be denoted by $A \bowtie H$. The left (resp. right) action $\triangleright: H \otimes A \rightarrow A$ (resp. $\triangleleft: H \otimes A \rightarrow H$ ) is called trivial if $y \triangleright a=\varepsilon_{H}(y) a$ (resp. $\left.y \triangleleft a=\varepsilon_{A}(a) y\right)$ for all $a \in A$ and $y \in H$.

The smash product of Hopf algebras (also called semi-direct product) appears as a special case of the bicrossed product construction. More precisely, if $(A, \triangleright)$ is a left $H$-module coalgebra and we consider $H$ as a right $A$-module coalgebra via the trivial action, i.e. $y \triangleleft a=\varepsilon_{A}(a) y$, then $(A, H, \triangleleft, \triangleright)$ is a matched pair of Hopf algebras if and only if $(A, \triangleright)$ is also a left $H$-module algebra and the following compatibility condition holds for all $y \in H$ and $a \in A$ :

$$
y_{(1)} \otimes y_{(2)} \triangleright a=y_{(2)} \otimes y_{(1)} \triangleright a .
$$

In this case, the associated bicrossed product $A \bowtie H=A \# H$ is the left version of the smash (semi-direct) product of Hopf algebras. Thus, $A \# H$ is the tensor coalgebra $A \otimes H$, with the following multiplication:

$$
(a \# y) \cdot(b \# z):=a\left(y_{(1)} \triangleright b\right) \# y_{(2)} z
$$

for all $a, b \in A, y, z \in H$, where we denote $a \otimes y$ by a\#y. Symmetrically, we can define the right version of the smash product of Hopf algebras by considering $A$ to be a left $H$-module coalgebra via the trivial action. 
Other important examples of bicrossed products include the classical Drinfel'd double ([13, Theorem IX.3.5]) as well as the generalized quantum double ([17, Example 7.2.6]). We refer the reader to [17] for more details and further examples.

Bicrossed products were introduced in connection to the factorization problem. More precisely, any Hopf algebra which factorizes through two given Hopf algebras is isomorphic to a bicrossed product between the same two Hopf algebras ([16]). Recall that the factorization problem for Hopf algebras consists of describing and classifying all Hopf algebras which factorize through two given Hopf algebras, i.e. all Hopf algebras for which there exist injective Hopf algebra maps $i: A \rightarrow E$ and $j: H \rightarrow E$ such that the map $A \otimes H \rightarrow E, \quad a \otimes y \mapsto i(a) j(y)$ is bijective.

\section{MaIN RESUlts}

In this section we investigate bicrossed products between the Taft algebra and the group Hopf algebra of certain groups or, equivalently, Hopf algebras which factorize through the aforementioned Hopf algebras. More precisely, our first result states that if the group $G$ admits a generating set consisting of finite order elements then any bicrossed product between the Taft algebra and $K[G]$ is in fact a smash product.

Theorem 2.1. Let $G$ be a group generated by finite order elements. Then any Hopf algebra which factorizes through the Taft algebra $T_{m^{2}}(q)$ and the group Hopf algebra $K[G]$ is isomorphic to a smash product between the same two Hopf algebras.

Proof. Consider $S$ to be a generating set of $G$ consisting of finite order elements and let $g \in S$. According to our assumption we have $\operatorname{ord}(g)=u$, where $u \in \mathbb{N}^{*}$.

Let $\left(T_{m^{2}}(q), K[G], \triangleleft, \triangleright\right)$ be a matched pair of Hopf algebras where $\triangleleft: K[G] \otimes T_{m^{2}}(q) \rightarrow K[G]$, $\rightarrow$ $K[G] \otimes T_{m^{2}}(q) \rightarrow T_{m^{2}}(q)$ are coalgebra maps satisfying the compatibility conditions (3)-(6). We will prove that the right action $\triangleleft$ is trivial. Indeed, by [3, Lemma 5.1] we have $g \triangleright h \in \mathcal{G}\left(T_{m^{2}}(q)\right)$, i.e. $g \triangleright h=h^{i}$ for some $i \in\{0,1, \cdots, m-1\}$. Assume first that $i=0$; by induction one obtains $1=g^{u} \triangleright h=h$ which is an obvious contradiction. Thus we have $i \in\{1,2, \cdots, m-1\}$. Suppose now that $i \neq 1$; in this case [3, Lemma 5.1] gives $g \triangleright x \in \mathcal{P}_{g \triangleright h, g \triangleright 1}\left(T_{m^{2}}(q)\right)=\mathcal{P}_{h^{i}, 1}\left(T_{m^{2}}(q)\right)$ and since $i \neq 1$ it follows from (2) that $g \triangleright x=\alpha\left(h^{i}-1\right)$ for some $\alpha \in K$. By induction we obtain $x=g^{u} \triangleright x=\alpha g^{u-1} \triangleright h^{i}-\alpha$ and we have reached a contradiction as [3, Lemma 5.1] implies $g^{u-1} \triangleright h^{i} \in \mathcal{G}\left(T_{m^{2}}(q)\right)$. Hence we are led to $g \triangleright h=h$. Furthermore, this implies $g \triangleright x=\alpha(1-h)+\beta x$ for some $\alpha, \beta \in K$. Again by induction we arrive at $x=g^{u} \triangleright x=\alpha\left(1+\beta+\cdots+\beta^{u-1}\right)(1-h)+\beta^{u} x$ which yields:

$$
\alpha\left(1+\beta+\cdots+\beta^{u-1}\right)=0, \quad \beta^{u}=1 .
$$

For the rest of the proof we will focus on the right action $\triangleleft$. Using [3, Lemma 5.1] we obtain $g \triangleleft h \in$ $\mathcal{G}(K[G])$. Remark that the case $g \triangleleft h=1$ can be easily ruled out by the same arguments used for the left action. Hence $g \triangleleft h=g_{i_{1}}^{a_{1}} g_{i_{2}}^{a_{2}} \cdots g_{i_{s}}^{a_{s}}$ for some $s, a_{1}, \cdots, a_{s} \in \mathbb{N}^{*}$ and $g_{i_{1}}, \cdots, g_{i_{s}} \in S$. Moreover, again by [3, Lemma 5.1] we have $g \triangleleft x \in \mathcal{P}_{g \triangleleft h, g \triangleleft 1}(K[G])=\mathcal{P}_{g_{i_{1}}^{a_{1}} g_{i_{2}} a_{2} \ldots g_{i_{s}}, g}(K[G])$, i.e. $g \triangleleft x=\gamma\left(g-g_{i_{1}}^{a_{1}} g_{i_{2}}^{a_{2}} \cdots g_{i_{s}}^{a_{s}}\right)$ for some $\gamma \in K$. Now we apply (6) for the pair $(g, x)$ and we obtain:

$$
g \triangleleft x \otimes h+g \otimes g \triangleright x=g \triangleleft h \otimes g \triangleright x+g \triangleleft x \otimes 1 .
$$

Furthermore, this gives:

$$
\gamma g \otimes h-\gamma g_{i_{1}}^{a_{1}} g_{i_{2}}^{a_{2}} \cdots g_{i_{s}}^{a_{s}} \otimes h+\alpha g \otimes 1-\alpha g \otimes h+\beta g \otimes x=
$$




$$
\alpha g_{i_{1}}^{a_{1}} g_{i_{2}}^{a_{2}} \cdots g_{i_{s}}^{a_{s}} \otimes 1-\alpha g_{i_{1}}^{a_{1}} g_{i_{2}}^{a_{2}} \cdots g_{i_{s}}^{a_{s}} \otimes h+\beta g_{i_{1}}^{a_{1}} g_{i_{2}}^{a_{2}} \cdots g_{i_{s}}^{a_{s}} \otimes x+\gamma g \otimes 1-\gamma g_{i_{1}}^{a_{1}} g_{i_{2}}^{a_{2}} \cdots g_{i_{s}}^{a_{s}} \otimes 1
$$

In particular, (8) implies $\beta g \otimes x=\beta g_{i_{1}}^{a_{1}} g_{i_{2}}^{a_{2}} \cdots g_{i_{s}}^{a_{s}} \otimes x$ and since by (7) we have $\beta \neq 0$ it follows that $g_{i_{1}}^{a_{1}} g_{i_{2}}^{a_{2}} \cdots g_{i_{s}}^{a_{s}}=g$. Therefore, we have $g \triangleleft h=g$ and $g \triangleleft x=0$.

Next, by using the compatibility condition (4) we arrive at the following:

$$
\begin{aligned}
& g \triangleright h x \stackrel{4}{=} \quad(g \triangleright h)((g \triangleleft h) \triangleright x)=h(g \triangleright x)=\alpha\left(h-h^{2}\right)+\beta h x \\
& g \triangleright x h \stackrel{4}{=} \quad\left(g \triangleright x_{(1)}\right)\left(\left(g \triangleleft x_{(2)}\right) \triangleright h\right)=(g \triangleright x)((g \triangleleft h) \triangleright h)+(g \triangleright 1)((g \triangleleft x) \triangleright h) \\
&=\quad \alpha\left(h-h^{2}\right)+\beta x h=\alpha\left(h-h^{2}\right)+\beta q h x
\end{aligned}
$$

As $x h=q h x$, using the above computation gives:

$$
q \alpha h-q \alpha h^{2}+q \beta h x=\alpha h-\alpha h^{2}+\beta x h
$$

and since $q \neq 1$ we obtain $\alpha=0$. Thus $g \triangleright x=\beta x$.

Analogously, one can prove that for any $g_{i} \in S$ with $\operatorname{ord}\left(g_{i}\right)=u_{i}$ we have:

$$
g_{i} \triangleright h=h, g_{i} \triangleright x=\beta_{i} x, \quad g_{i} \triangleleft h=g_{i}, \quad g_{i} \triangleleft x=0, \text { where } \beta_{i} \in U_{u_{i}}(K) .
$$

We are now ready to prove that the right action $\triangleleft: K[G] \otimes T_{m^{2}}(q) \rightarrow K[G]$ is indeed trivial. To this end, for all $g_{i} \in S$ we have:

$$
g_{i}^{2} \triangleleft h \stackrel{5]}{=}\left(g_{i} \triangleleft\left(g_{i} \triangleleft h\right)\right)\left(g_{i} \triangleleft h\right)=\left(g_{i} \triangleleft h\right)\left(g_{i} \triangleleft h\right)=g_{i}^{2}
$$

and by induction one can readily see that for all $v \in\left\{0,1, \cdots, u_{i}-1\right\}$ we have $g_{i}^{v} \triangleleft h=g_{i}^{v}$. Hence, we obtain:

$$
g_{i}^{v} \triangleleft h^{j}=g_{i}^{v}, \text { for all } v \in\left\{0,1, \cdots, u_{i}-1\right\} \text { and } j \in\{0,1, \cdots, m-1\} .
$$

Similarly, using (4), yields:

$$
g_{i}^{v} \triangleright h^{j}=h^{j}, \text { for all } v \in\left\{0,1, \cdots, u_{i}-1\right\} \text { and } j \in\{0,1, \cdots, m-1\} .
$$

By putting together (10) and (11) gives:

$$
g_{i_{1}}^{\nu_{1}} g_{i_{2}}^{\nu_{2}} \triangleleft h^{j} \stackrel{\text { 5] }}{=}\left(g_{i_{1}}^{\nu_{1}} \triangleleft\left(g_{i_{2}}^{\nu_{2}} \triangleleft h^{j}\right)\right)\left(g_{i_{2}}^{\nu_{2}} \triangleleft h^{j}\right) \stackrel{11}{=}\left(g_{i_{1}}^{\nu_{1}} \triangleleft h^{j}\right)\left(g_{i_{2}}^{\nu_{2}} \triangleleft h^{j}\right) \stackrel{10}{=} g_{i_{1}}^{\nu_{1}} g_{i_{2}}^{\nu_{2}}
$$

for all $g_{i_{1}}, g_{i_{2}} \in S, v_{1} \in\left\{0,1, \cdots, u_{i_{1}}-1\right\}, v_{2} \in\left\{0,1, \cdots, u_{i_{2}}-1\right\}$ and $j \in\{0,1, \cdots, m-1\}$. Inductively, we obtain:

$$
g_{i_{1}}^{v_{1}} g_{i_{2}}^{\nu_{2}} \cdots g_{i_{t}}^{v_{t}} \triangleleft h^{j}=g_{i_{1}}^{\nu_{1}} g_{i_{2}}^{\nu_{2}} \cdots g_{i_{t}}^{v_{t}}
$$

for all $g_{i_{l}} \in S, v_{l} \in\left\{0,1, \cdots, u_{i_{l}}-1\right\}, l \in\{1,2, \cdots, t\}$ and $j \in\{0,1, \cdots, m-1\}$. Furthermore, we also have:

$$
g_{i}^{2} \triangleleft x \stackrel{5}{=}\left(g_{i} \triangleleft\left(g_{i} \triangleright x_{(1)}\right)\right)\left(g_{i} \triangleleft x_{(2)}\right)=\left(g_{i} \triangleleft\left(g_{i} \triangleright x\right)\right)\left(g_{i} \triangleleft h\right)+\left(g_{i} \triangleleft\left(g_{i} \triangleright 1\right)\right)\left(g_{i} \triangleleft x\right)=0
$$

and using induction again we get $g_{i}^{v} \triangleleft x^{j}=0$ for all $v \in\left\{0,1, \cdots, u_{i}-1\right\}$ and $j \in\{1,2, \cdots, m-1\}$. Now remark that we actually proved that:

$$
g_{i}^{v} \triangleleft x^{j}=g_{i}^{v} \varepsilon\left(x^{j}\right), \text { for all } v \in\left\{0,1, \cdots, u_{i}-1\right\} \text { and } j \in\{0,1, \cdots, m-1\} \text {. }
$$

As before, one can easily prove that:

$$
g_{i_{1}}^{\nu_{1}} g_{i_{2}}^{\nu_{2}} \cdots g_{i_{t}}^{\nu_{t}} \triangleleft x^{j}=g_{i_{1}}^{\nu_{1}} g_{i_{2}}^{\nu_{2}} \cdots g_{i_{t}}^{\nu_{t}} \varepsilon\left(x^{j}\right)
$$


for all $g_{i_{l}} \in S, v_{l} \in\left\{0,1, \cdots, u_{i_{l}}-1\right\}, l \in\{1,2, \cdots, t\}$ and $j \in\{0,1, \cdots, m-1\}$. Finally, by putting all the above together yields:

$$
\left.g_{i_{1}}^{v_{1}} g_{i_{2}}^{v_{2}} \cdots g_{i_{t}}^{v_{t}} \triangleleft h^{j} x^{j^{\prime}}=\left(g_{i_{1}}^{v_{1}} g_{i_{2}}^{v_{2}} \cdots g_{i_{t}}^{v_{t}} \triangleleft h^{j}\right) \triangleleft x^{j^{\prime}} \stackrel{12}{=}, 114\right) g_{i_{1}}^{v_{1}} g_{i_{2}}^{v_{2}} \cdots g_{i_{t}}^{v_{t}} \varepsilon\left(x^{j^{\prime}}\right)=g_{i_{1}}^{v_{1}} g_{i_{2}}^{v_{2}} \cdots g_{i_{t}}^{v_{t}} \varepsilon\left(h^{j} x^{j^{\prime}}\right)
$$

for all $g_{i_{l}} \in S, v_{l} \in\left\{0,1, \cdots, u_{i_{l}}-1\right\}, l \in\{1,2, \cdots, t\}, j, j^{\prime} \in\{0,1, \cdots, m-1\}$, i.e. the right action is indeed trivial and the proof is now finished.

Notice that although Theorem 2.1 shows that the right action of any bicrossed product between the Taft algebra $T_{m^{2}}(q)$ and the group Hopf algebra $K[G]$ is trivial whenever $G$ admits a generating set consisting of finite order elements, the description of the left action is only narrow as it depends essentially on the relations, if any, satisfied by the generators of the group $G$. In order to illustrate this idea, in the sequel we will focus on the case where $G=D_{l n}^{k}$ as defined in (1). We will be able to describe completely by generators and relations all Hopf algebras which factorize through the Taft algebra and the group Hopf algebra $K\left[D_{l n}^{k}\right]$. We start by computing all matched pairs $\left(T_{m^{2}}(q), K\left[D_{l n}^{k}\right], \triangleleft, \triangleright\right)$ : as a consequence of Theorem 2.1 the right action is trivial while the left action is implemented by two roots of unity $\beta \in U_{l}(K), \sigma \in U_{(n, k-1)}(K)$.

Theorem 2.2. There exists a bijection between the set of all matched pairs $\left(T_{m^{2}}(q), K\left[D_{l n}^{k}\right], \triangleleft, \triangleright\right)$ and $U_{l}(K) \times U_{(n, k-1)}(K)$ such that the matched pair $(\triangleleft, \triangleright)$ corresponding to $(\beta, \sigma) \in U_{l}(K) \times U_{(n, k-1)}(K)$ is given by:

$$
d^{t} c^{i} \triangleright h^{j^{\prime}} x^{j}=\beta^{i j} \sigma^{t j} h^{j^{\prime}} x^{j}, \quad d^{t} c^{i} \triangleleft h^{j^{\prime}} x^{j}=d^{t} c^{i} \varepsilon\left(h^{j^{\prime}} x^{j}\right)
$$

for all $t=0, \cdots, n-1, i=0, \cdots, l-1$ and $j, j^{\prime}=0, \cdots, m-1$.

Furthermore, any bicrossed product between $T_{m^{2}}(q)$ and $K\left[D_{l n}^{k}\right]$ is isomorphic to $T_{\operatorname{lnm}^{2}}^{\beta, \sigma}(q)$, for some $(\beta, \sigma) \in U_{l}(K) \times U_{(n, k-1)}(K)$, where $T_{l n m^{2}}^{\beta, \sigma}(q)$ is the Hopf algebra generated by $c, d$, $h$ and $x$ subject to the following relations:

$$
\begin{gathered}
c^{l}=d^{n}=h^{m}=1, \quad x^{m}=0, \\
c h=h c, \quad d h=h d, \quad c d=d^{k} c, \\
c x=\beta x c, \quad d x=\sigma x d, \quad x h=q h x
\end{gathered}
$$

with the coalgebra structure and antipode given by:

$$
\begin{array}{cl}
\Delta(c)=c \otimes c, \quad \Delta(d)=d \otimes d, \quad \Delta(h)=h \otimes h, \quad \Delta(x)=x \otimes h+1 \otimes x, & \varepsilon(x)=0, \\
\varepsilon(a)=\varepsilon(b)=\varepsilon(h)=1, \quad S(h)=h^{m-1}, \quad S(x)=-x h^{m-1}, \quad S(c)=c^{l-1}, & S(d)=d^{n-1} .
\end{array}
$$

Proof. Let $\left(T_{m^{2}}(q), K\left[D_{l n}^{k}\right], \triangleleft, \triangleright\right)$ be a matched pair. Since the group $D_{l n}^{k}$ is generated by two finite order elements, namely $c$ and $d$, we can conclude by (the proof of) Theorem 2.1 that the right action is trivial and the left action is given as follows for all $i \in\{0,1, \cdots, l-1\}$ and $j \in\{0,1, \cdots, n-1\}$ :

$$
c^{i} \triangleright h=h, c^{i} \triangleright x=\beta^{i} x, d^{j} \triangleright h=h, d^{j} \triangleright x=\sigma^{j} x \text { where } \beta \in U_{l}(K), \sigma \in U_{n}(K) .
$$

Furthermore, since the relation $c d c^{l-1}=d^{k}$ holds in $D_{l n}^{k}$ we have $c d c^{l-1} \triangleright x=d^{k} \triangleright x$ which after a straightforward computation gives $\sigma x=\sigma^{k} x$, i.e. $\tau \in U_{k-1}(K)$. Therefore we obtain $\sigma \in U_{(n, k-1)}(K)$ as desired. Finally, the formulae in (15) can be easily derived using the defining compatibility conditions of a matched pair of Hopf algebras (i.e. (3) - (6) ) exactly as in the proof of Theorem 2.1.

Thus any bicrossed product between $T_{m^{2}}(q)$ and $K\left[D_{l n}^{k}\right]$ is in fact a smash product $T_{m^{2}}(q) \# K\left[D_{l n}^{k}\right]$ associated to the left action determined by a pair $(\beta, \sigma) \in U_{l}(K) \times U_{(n, k-1)}(K)$ as in (16). Up to 
canonical identification, $T_{m^{2}}(q) \# K\left[C_{n}\right]$ is generated as an algebra by $h=h \# 1, x=x \# 1, c=1 \# c$ and $d=1 \# d$. For instance, we have:

$$
\begin{aligned}
& c h=(1 \# c)(h \# 1)=(c \triangleright h) \#(c \triangleleft h)=h \# c=(h \# 1)(1 \# c)=h c \\
& c x=(1 \# c)(x \# 1)=\left(a \triangleright x_{(1)}\right) \#\left(c \triangleleft x_{(2)}\right)=(c \triangleright x) \#(c \triangleleft h)+(c \triangleright 1) \#(c \triangleleft x)=\beta x \# c=\beta x c
\end{aligned}
$$

Since the rest of the defining relations of $T_{\operatorname{lnm} m^{2}}^{\beta, \sigma}(q)$ can be checked in much the same fashion as above they are left to the reader.

Our next goal is to classify the Hopf algebras described in Theorem 2.1. We will see that actually the quest for classifying all bicrossed products between the Taft algebra and the group Hopf algebra $K[G]$, where $G$ is a group generated by a set of finite order elements, comes down to an old and notoriously difficult problem in group theory, namely that of describing the automorphisms of a certain given group. More precisely, our next result shows that any Hopf algebra isomorphism between two smash products as in Theorem 2.1 is in some sense induced by a group automorphism of $G$.

Theorem 2.3. Let $G$ be a group generated by a set of finite order elements and consider two smash products $T_{m^{2}}(q) \# K[G]$ and $T_{m^{2}}(q) \#^{\prime} K[G]$. If $\varphi: T_{m^{2}}(q) \# K[G] \rightarrow T_{m^{2}}(q) \#^{\prime} K[G]$ is a Hopf algebra isomorphism then there exist two unitary coalgebra maps $u: T_{m^{2}}(q) \rightarrow T_{m^{2}}(q), r: K[G] \rightarrow$ $T_{m^{2}}(q)$ and a Hopf algebra automorphism $v: K[G] \rightarrow K[G]$ such that $\varphi=\varphi_{(u, r, v)}$, where $\varphi_{(u, r, v)}$ : $T_{m^{2}}(q) \# K[G] \rightarrow T_{m^{2}}(q) \#^{\prime} K[G]$ is given as follows for all $a \in T_{m^{2}}(q)$ and $t \in K[G]:$

$$
\varphi_{(u, r, v)}(a \# t)=u(a) r\left(t_{(1)}\right) \#^{\prime} v\left(t_{(2)}\right) .
$$

Proof. Consider $S=\left\{g_{i} \in G \mid i \in I\right\}$ to be a generating set of $G$ with $\operatorname{ord}\left(g_{i}\right)=u_{i}$, where $u_{i} \in \mathbb{N}^{*}$ for all $i \in I$. According to Theorem 2.1, the left actions $\triangleright: K[G] \otimes T_{m^{2}}(q) \rightarrow T_{m^{2}}(q), \triangleright^{\prime}: K[G] \otimes T_{m^{2}}(q) \rightarrow$ $T_{m^{2}}(q)$, corresponding to the two smash products $T_{m^{2}}(q) \# K[G]$ and $T_{m^{2}}(q) \#^{\prime} K[G]$, respectively, are given as follows for all $i \in I$ :

$$
g_{i} \triangleright h=h, \quad g_{i} \triangleright x=\alpha_{i} x, \quad g_{i} \triangleright^{\prime} h=h, \quad g_{i} \triangleright^{\prime} x=\bar{\alpha}_{i} x, \text { where } \alpha_{i}, \bar{\alpha}_{i} \in U_{u_{i}}(K) .
$$

By [3, Theorem 3.2], the set of all Hopf algebra morphisms between $T_{m^{2}}(q) \# K[G]$ and $T_{m^{2}}(q) \#^{\prime} K[G]$ is in bijective correspondence with the set of all quadruples $(u, p, r, v)$, consisting of two unitary coalgebra maps $u: T_{m^{2}}(q) \rightarrow T_{m^{2}}(q)$ and $r: K[G] \rightarrow T_{m^{2}}(q)$, and two Hopf algebra maps $p: T_{m^{2}}(q) \rightarrow K[G]$ and $v: K[G] \rightarrow K[G]$ subject to the following compatibilities for all $a, b \in T_{m^{2}}(q), t, w \in K[G]$ :

$$
\begin{aligned}
u\left(a_{(1)}\right) \otimes p\left(a_{(2)}\right) & =u\left(a_{(2)}\right) \otimes p\left(a_{(1)}\right) \\
u(a b) & =u\left(a_{(1)}\right)\left(p\left(a_{(2)}\right) \triangleright^{\prime} u(b)\right) \\
r(t w) & =r\left(t_{(1)}\right)\left(v\left(t_{(2)}\right) \triangleright^{\prime} r(w)\right) \\
r\left(t_{(1)}\right)\left(v\left(t_{(2)}\right) \triangleright^{\prime} u(a)\right) & =u\left(t_{(1)} \triangleright a_{(1)}\right)\left(p\left(t_{(2)} \triangleright a_{(2)}\right) \triangleright^{\prime} r\left(t_{(3)}\right)\right) \\
v(t) p(a) & =p\left(t_{(1)} \triangleright a\right) v\left(t_{(2)}\right.
\end{aligned}
$$

The correspondence is such that the morphism $\psi_{(u, p, r, v)}$ associated to $(u, p, r, v)$ is given as follows for all $a \in T_{m^{2}}(q)$ and $t \in K[G]$ :

$$
\psi_{(u, p, r, v)}(a \# t)=u\left(a_{(1)}\right)\left(p\left(a_{(2)}\right) \triangleright^{\prime} r\left(t_{(1)}\right)\right) \#^{\prime} p\left(a_{(3)}\right) v\left(t_{(2)}\right) .
$$

We start by proving that the Hopf algebra morphism $p: T_{m^{2}}(q) \rightarrow K[G]$ of any quadruple $(u, p, r, v)$ satisfying the compatibilities (17) - (21) is trivial, i.e. $p=\varepsilon$. Indeed, since $p(h) \in \mathcal{G}(K[G])$ we have 
$p(h)=g_{i_{1}}^{a_{1}} g_{i_{2}}^{a_{2}} \cdots g_{i_{s}}^{a_{s}}$ for some $s, a_{1}, \cdots, a_{s} \in \mathbb{N}$ and $g_{i_{1}}, \cdots, g_{i_{s}} \in S$. Furthermore, this implies that $p(x) \in \mathcal{P}_{p(h), 1}(K[G])$, i.e. $p(x)=\beta\left(g_{i_{1}}^{a_{1}} g_{i_{2}}^{a_{2}} \cdots g_{i_{s}}^{a_{s}}-1\right)$ for some $\beta \in K$. Hence, we have:

$$
p(h x)=p(h) p(x)=\beta g_{i_{1}}^{a_{1}} g_{i_{2}}^{a_{2}} \cdots g_{i_{s}}^{a_{s}}\left(g_{i_{1}}^{a_{1}} g_{i_{2}}^{a_{2}} \cdots g_{i_{s}}^{a_{s}}-1\right)=\beta\left(g_{i_{1}}^{a_{1}} g_{i_{2}}^{a_{2}} \cdots g_{i_{s}}^{a_{s}}-1\right) g_{i_{1}}^{a_{1}} g_{i_{2}}^{a_{2}} \cdots g_{i_{s}}^{a_{s}}=p(x h) .
$$

As $p(x h)=q p(h x)$ we obtain $\beta(q-1) g_{i_{1}}^{a_{1}} g_{i_{2}}^{a_{2}} \cdots g_{i_{s}}^{a_{s}}\left(g_{i_{1}}^{a_{1}} g_{i_{2}}^{a_{2}} \cdots g_{i_{s}}^{a_{s}}-1\right)=0$ and since $q$ is a primitive $m$-th root of unity we have $q \neq 1$ and all the above comes down to $\beta\left(g_{i_{1}}^{a_{1}} g_{i_{2}}^{a_{2}} \cdots g_{i_{s}}^{a_{s}}-1\right)=0$, i.e. $p(x)=0$.

Next we investigate the unitary coalgebra maps $u: T_{m^{2}}(q) \rightarrow T_{m^{2}}(q)$. Firstly, we obviously have $u(1)=1$ and $u(h)=h^{d}$, where $d \in\{0,1, \cdots, m-1\}$. Now the compatibility condition (18) gives:

$$
u\left(h^{2}\right)=u(h)\left(p(h) \triangleright^{\prime} u(h)\right)=h^{d}\left(g_{i_{1}}^{a_{1}} g_{i_{2}}^{a_{2}} \cdots g_{i_{s}}^{a_{s}} \triangleright^{\prime} h^{d}\right)=h^{2 d} .
$$

By repeatedly applying (18) one easily obtains $u\left(h^{j}\right)=h^{j d}$ for any $j \in \mathbb{N}$. Furthermore, by using [3, Lemma 5.1], we have $u(x) \in \mathcal{P}_{h^{d}, 1}\left(T_{m^{2}}(q)\right)$ which leads to $u(x)=\mu\left(h^{d}-1\right)$ for some $\alpha \in K$ if $d \neq 1$ or to $u(x)=\mu(h-1)+\gamma x$ for some $\mu, \gamma \in K$ in the case when $d=1$.

Assume first that $d \neq 1$; thus $u(x)=\mu\left(h^{d}-1\right)$ and the compatibility condition (18) yields:

$$
\begin{aligned}
& u(h x) \stackrel{18}{\stackrel{18}{=}} u(h)\left(p(h) \triangleright^{\prime} u(x)\right)=h^{d}\left(g_{i_{1}}^{a_{1}} g_{i_{2}}^{a_{2}} \cdots g_{i_{s}}^{a_{s}} \triangleright^{\prime} \mu\left(h^{d}-1\right)\right)=\mu h^{d}\left(h^{d}-1\right) \\
& u(x h) \stackrel{18}{=} u\left(x_{(1)}\right)\left(p\left(x_{(2)}\right) \triangleright^{\prime} u(h)\right)=u(x)\left(p(h) \triangleright^{\prime} u(h)\right)+p(x) \triangleright^{\prime} u(h)=\mu h^{d}\left(h^{d}-1\right) .
\end{aligned}
$$

Now since $u(x h)=q u(h x)$ the above computations amount to $\mu(q-1) h^{d}\left(h^{d}-1\right)=0$. Moreover, as $q \neq 1$ we obtain $\mu\left(h^{d}-1\right)=0$ and thus $u(x)=0$. However, this contradicts the fact that the corresponding Hopf algebra map $\psi_{(u, p, r, v)}$ was assumed to be an isomorphism. Indeed we have:

$$
\psi_{(u, p, r, v)}(x \# 1)=u\left(x_{(1)}\right)\left(p\left(x_{(2)}\right) \triangleright^{\prime} r(1)\right) \# p\left(x_{(3)}\right) v(1)=u\left(x_{(1)}\right) \# p\left(x_{(2)}\right)=u(x) \# p(h)+1 \# p(x)=0 .
$$

Therefore the only possibility left is $d=1$ and $u(x)=\mu(h-1)+\gamma x$. In this case, we obtain:

$$
u(h x) \stackrel{18}{=} u(h)\left(p(h) \triangleright^{\prime} u(x)\right)=h\left[g_{i_{1}}^{a_{1}} g_{i_{2}}^{a_{2}} \cdots g_{i_{s}}^{a_{s}} \triangleright^{\prime}(\mu(h-1)+\gamma x)\right]=\mu h(h-1)+\gamma \alpha_{i_{1}}^{a_{1}} \alpha_{i_{2}}^{a_{2}} \cdots \alpha_{i_{s}}^{a_{s}} h x .
$$

A similar computation gives $u(x h)=\mu h(h-1)+\gamma q h x$ and since $u(x h)=q u(h x)$ we obtain $\mu=q \mu$ and $q \gamma \alpha_{i_{1}}^{a_{1}} \alpha_{i_{2}}^{a_{2}} \cdots \alpha_{i_{s}}^{a_{s}}=q \gamma$. As $q \neq 1$ we must have $\mu=0$. Thus $\gamma \neq 0$ (otherwise we would have $u(x)=0$ which leads to the same contradiction as in the case where $d \neq 1$ ) and so $\alpha_{i_{1}}^{a_{1}} \alpha_{i_{2}}^{a_{2}} \cdots \alpha_{i_{s}}^{a_{s}}=1$. Consequently, $u$ takes the following form:

$$
u\left(h^{i}\right)=h^{i}, u\left(x^{i}\right)=\gamma^{i} x^{i}, \quad \gamma \in K^{*}, \quad i \in\{0,1, \cdots, m-1\} .
$$

We are now in a position to prove that the Hopf algebra map $p: T_{m^{2}}(q) \rightarrow K[G]$ is indeed trivial. To this end, by setting $a=x$ the compatibility condition (17) reduces to the following:

$$
\begin{array}{ll} 
& u\left(x_{(1)}\right) \otimes p\left(x_{(2)}\right)=u\left(x_{(2)}\right) \otimes p\left(x_{(1)}\right) \\
\Leftrightarrow & u(x) \otimes p(h)+u(1) \otimes p(x)=u(h) \otimes p(x)+u(x) \otimes p(1) \\
\Leftrightarrow & \gamma x \otimes g_{i_{1}}^{a_{1}} g_{i_{2}}^{a_{2}} \cdots g_{i_{s}}^{a_{s}}=\gamma x \otimes 1 .
\end{array}
$$

Now recall that $\gamma \neq 0$ which leads to $g_{i_{1}}^{a_{1}} g_{i_{2}}^{a_{2}} \cdots g_{i_{s}}^{a_{s}}=1$ and thus $p$ is the trivial map, i.e. $p(a)=\varepsilon(a) 1$ for all $a \in T_{m^{2}}(q)$. Hence, the Hopf algebra isomorphism corresponding to the quadruple $(u, \varepsilon, r, v)$ as in (22) takes the following simplified form for all $a \# t \in T_{m^{2}}(q) \# K[G]$ :

$$
\psi_{(u, \varepsilon, r, v)}(a \# t)=u(a) r\left(t_{(1)}\right) \#^{\prime} v\left(t_{(2)}\right) .
$$


We are left to prove that $v$ is a Hopf algebra automorphism. Indeed, consider $\varphi: T_{m^{2}}(q) \bowtie^{\prime} K[G] \rightarrow$ $T_{m^{2}}(q) \bowtie K[G]$ to be the inverse of $\psi_{(u, \varepsilon, r, v)}$. Using the same arguments as above we can conclude that there exists a quadruple $(\bar{u}, \varepsilon, \bar{r}, \bar{v})$ as in (22) such that $\varphi=\psi_{(\bar{u}, \varepsilon, \bar{r}, \bar{v})}$, i.e.:

$$
\psi_{(\bar{u}, \varepsilon, \bar{r}, \bar{v})}\left(a \#^{\prime} t\right)=\bar{u}(a) \bar{r}\left(t_{(1)}\right) \# \bar{v}\left(t_{(2)}\right) \text {, for all } a \#^{\prime} t \in T_{m^{2}}(q) \#^{\prime} K[G] .
$$

Then, for any $t \in K[G]$ we have:

$$
1 \#^{\prime} t=\psi_{(u, \varepsilon, r, v)} \circ \psi_{(\bar{u}, \varepsilon, \bar{r}, \bar{v})}\left(1 \#^{\prime} t\right)=\psi_{(u, \varepsilon, r, v)}\left(\bar{r}\left(t_{(1)}\right) \# \bar{v}\left(t_{(2)}\right)\right)=u\left(\bar{r}\left(t_{(1)}\right)\right) r\left(\bar{v}\left(t_{(2)}\right)\right) \#^{\prime} v\left(\bar{v}\left(t_{(3)}\right)\right) \text {. }
$$

By applying $\varepsilon \otimes$ Id to the above identity yields $v \circ \bar{v}(t)=t$ for any $t \in K[G]$. Similarly one can prove that $\bar{v} \circ v(t)=t$ and the proof is now finished.

Obviously, the Hopf algebra automorphism $v: K[G] \rightarrow K[G]$ from Theorem 2.3 is uniquely determined by a group automorphism of $G$. In the sequel we will provide a complete classification for the Hopf algebras which factorize through the Taft algebra and the group Hopf algebra $K\left[D_{2 n}\right]$. To this end, recall that for any $n>2$ we have $\operatorname{Aut}\left(D_{2 n}\right)=\left\{\gamma_{s, t} \mid s, t=0,1, \cdots, n-1,(t, n)=1\right\}$, where $\gamma_{s, t}: D_{2 n} \rightarrow D_{2 n}$ is the group automorphism defined as follows:

$$
\gamma_{s, t}(c)=d^{s} c, \quad \gamma_{s, t}(d)=d^{t} .
$$

Consider $\beta, \bar{\beta} \in U_{2}(K), \sigma, \bar{\sigma} \in U_{(n, n-2)}(K)$ and let $T_{2 n m^{2}}^{\beta, \sigma}(q)$ and $T_{2 n m^{2}}^{\bar{\beta}, \bar{\sigma}}(q)$, respectively, to be the corresponding Hopf algebras as in Theorem 2.2. Our next result provides necessary and sufficient conditions for the two aforementioned Hopf algebras to be isomorphic.

Theorem 2.4. Let $m, n \in \mathbb{N}^{*}, m \geq 2$ and $n \geq 3$. Then the Hopf algebras $T_{2 n m^{2}}^{\beta, \sigma}(q)$ and $T_{2 n m^{2}}^{\bar{\beta}, \bar{q}}(q)$ are isomorphic if and only if there exist integers $f, F \in\{0,1, \cdots, m-1\}$ and $s, t \in\{0,1, \cdots, n-1\}$ such that $(t, n)=1$ and the following compatibilities hold:

$$
\begin{gathered}
m|2 f, \quad m| n F, \quad m \mid 2 F, \\
q^{f}=\beta \bar{\beta} \bar{\sigma}^{s}, \quad q^{F}=\sigma \bar{\sigma}^{t} .
\end{gathered}
$$

Proof. In light of (the proof of) Theorem 2.3 the Hopf algebras $T_{2 n m^{2}}^{\beta, \sigma}(q)$ and $T_{2 n m^{2}}^{\bar{\beta}, \bar{\sigma}}(q)$ are isomorphic if and only if there exists a quadruple $(u, p, r, v)$ satisfying the compatibilities (17)-(21) such that the corresponding morphism $\psi_{(u, p, r, v)}: T_{2 n m^{2}}^{\beta, \sigma}(q) \rightarrow T_{2 n m^{2}}^{\bar{\beta}, \bar{\sigma}}(q)$ given by (22) is an isomorphism. It follows from Theorem 2.3 that $\psi_{(u, p, r, v)}$ being an isomorphism implies $p=\varepsilon$ and $v \in \operatorname{Aut}_{\mathrm{Hopf}}\left(K\left[D_{2 n}\right]\right)$. Furthermore, as $p$ is the trivial map, the compatibility conditions (17) and (21) are trivially fulfilled while (18) comes down to $u: T_{m^{2}}(q) \rightarrow T_{m^{2}}(q)$ being a Hopf algebra morphism. More precisely, exactly as in (the proof of) Theorem [2.3, we can conclude that $u$ is given as follows for all $i, j \in$ $\{0,1, \cdots, m-1\}$ :

$$
u\left(h^{i} x^{j}\right)=\gamma^{j} h^{i} x^{j}, \text { where } \gamma \in K^{*} .
$$

We are left to discuss the compatibility conditions (19) and (20). To start with, the Hopf algebra automorphism $v: K\left[D_{2 n}\right] \rightarrow K\left[D_{2 n}\right]$ and the coalgebra map $r: K\left[D_{2 n}\right] \rightarrow T_{m^{2}}(q)$ are uniquely determined by some integers $s, t \in\{0,1, \cdots, n-1\}, f, F \in\{0,1, \cdots, m-1\}$, such that $(t, n)=1$ and:

$$
v(c)=d^{s} c, \quad v(d)=d^{t} \quad \text { and } \quad r(c)=h^{f}, \quad r(d)=h^{F} .
$$


Using the compatibility condition (19) yields:

$$
r\left(c^{2}\right) \stackrel{19}{=} r(c)\left(v(c) \triangleright^{\prime} r(c)\right)=h^{f}\left(d^{s} c \triangleright^{\prime} h^{f}\right)=h^{2 f}
$$

which implies $m \mid 2 f$. Similarly, using induction and (19) we obtain $r\left(d^{i}\right)=h^{i F}$ for all $i \in \mathbb{N}$. In particular, we get $1=r\left(d^{n}\right)=h^{n F}$ and thus $m \mid n F$. Furthermore, we have:

$$
\begin{aligned}
r(c d) \stackrel{19}{=} r(c)\left(v(c) \triangleright^{\prime} r(d)\right)=h^{f}\left(d^{s} c \triangleright^{\prime} h^{F}\right)=h^{f+F} \\
r\left(d^{n-1} c\right) \stackrel{19}{=} r\left(d^{n-1}\right)\left(v\left(d^{n-1}\right) \triangleright^{\prime} r(c)\right)=h^{(n-1) F}\left(\left(d^{t}\right)^{n-1} \triangleright^{\prime} h^{f}\right)=h^{(n-1) F+f}=h^{-F+f} .
\end{aligned}
$$

Therefore we obtain $h^{f+F}=h^{-F+f}$ which leads to $m \mid 2 F$ as desired. Next we look at the compatibility condition (20); as $p$ is the trivial map it comes down to the following:

$$
r\left(t_{(1)}\right)\left(v\left(t_{(2)}\right) \triangleright^{\prime} u(a)\right)=u\left(t_{(1)} \triangleright a\right) r\left(t_{(2)}\right) .
$$

By considering $t=c$ and $a=x$ in 25 yields:

$$
\begin{aligned}
& r(c)\left(v(c) \triangleright^{\prime} u(x)\right)=u(c \triangleright x) r(c) \\
\Leftrightarrow & h^{f}\left(d^{s} c \triangleright^{\prime} \gamma x\right)=\gamma \beta x h^{f} \\
\Leftrightarrow & \gamma \bar{\beta} \bar{\sigma}^{s} h^{f} x=\gamma \beta q^{f} h^{f} x .
\end{aligned}
$$

Hence we have $q^{f}=\beta \bar{\beta} \bar{\sigma}^{s}$. Similarly, by setting $t=d$ and $a=x$ in (25) gives $q^{F}=\sigma \bar{\sigma}^{t}$.

Putting all together, we proved that if $T_{2 n m^{2}}^{\beta, \sigma}(q)$ and $T_{2 n m^{2}}^{\bar{\beta}, \bar{\sigma}}(q)$ are isomorphic Hopf algebras then there exist integers $s, t \in\{0,1, \cdots, n-1\}$ and $f, F \in\{0,1, \cdots, m-1\}$ with $(t, n)=1$ such that (23) and (24) are fulfilled.

Conversely, if there exist $s, t \in\{0,1, \cdots, n-1\}$ and $f, F \in\{0,1, \cdots, m-1\}$ satisfying the conditions above, we construct a coalgebra map $r_{(f, F)}: K\left[D_{2 n}\right] \rightarrow T_{m^{2}}(q)$ and a Hopf algebra automorphism $v_{(s, t)}: K\left[D_{2 n}\right] \rightarrow K\left[D_{2 n}\right]$ as follows:

$$
r_{(f, F)}\left(d^{i} c^{j}\right)=h^{i F+j f}, \quad v_{(s, t)}(c)=d^{s} c, \quad v_{(s, t)}(d)=d^{t} \quad \text { for } \quad \text { all } \quad i, j \in \mathbb{N} .
$$

Then it can be readily proved that the following map is a Hopf algebra isomorphism:

$$
\varphi:=\psi_{\left(\mathrm{Id}, \varepsilon, r_{(f, F)}, v_{(s, t)}\right)}: T_{2 n m^{2}}^{\beta, \sigma}(q) \rightarrow T_{2 n m^{2}}^{\bar{\beta}, \bar{\sigma}}(q), \quad \varphi(a \# y)=\operatorname{ar}_{(f, F)}\left(y_{(1)}\right) \# v_{(s, t)}\left(y_{(2)}\right) .
$$

where Id $: T_{m^{2}}(q) \rightarrow T_{m^{2}}(q)$ is the identity map on $T_{m^{2}}(q)$. First notice that the first part of the proof implies that $\varphi$ is a Hopf algebra map. We are left to prove that $\varphi$ is in fact an isomorphism. To start with, since $(t, n)=1$ there exist $\tau, \tau^{\prime} \in \mathbb{Z}$ such that $t \tau+n \tau^{\prime}=1$. Moreover, we can find unique integers $\alpha, \beta, \eta, \mu \in \mathbb{Z}, \bar{f}, \bar{F} \in\{0,1, \cdots, m-1\}$ and $\tau_{1}, \tau_{2} \in\{0,1, \cdots, n-1\}$ such that:

$$
\begin{aligned}
\tau & =\eta n+\tau_{1}, & & -\tau_{1} s=\mu n+\tau_{2}, \\
-f-F \tau_{2} & =\alpha m+\bar{f}, & & -F \tau_{1}=\beta m+\bar{F} .
\end{aligned}
$$

We are now in a position to construct the inverse of $\varphi$. To this end, let $r_{(\bar{f}, \bar{F})}: K\left[D_{2 n}\right] \rightarrow T_{m^{2}}(q)$ be a coalgebra map and $v_{\left(\tau_{2}, \tau_{1}\right)}: K\left[D_{2 n}\right] \rightarrow K\left[D_{2 n}\right]$ a Hopf algebra map, defined as follows:

$$
r_{\overline{(f}, \bar{F})}\left(d^{i} c^{j}\right)=h^{i \bar{F}+\bar{j}}, \quad v_{\left(\tau_{2}, \tau_{1}\right)}(c)=d^{\tau_{2}} c, \quad v_{\left(\tau_{2}, \tau_{1}\right)}(d)=d^{\tau_{1}} \quad \text { for } \quad \text { all } \quad i, j \in \mathbb{N} .
$$


The proof will be finished once we prove that the map $\bar{\varphi}:=\psi_{\left(\mathrm{Id}, \varepsilon, r_{\bar{f}, \bar{F}}, v_{\left(\tau_{2}, \tau_{1}\right)}\right)}$ is the inverse of $\varphi$. Indeed, for instance we have:

$$
\begin{aligned}
\varphi \circ \bar{\varphi}\left(h^{i} x^{j} \# d^{k}\right)= & \varphi\left(h^{i} x^{j} r_{\bar{f}}, \bar{F}\left(d^{k}\right) \# v_{\left(\tau_{2}, \tau_{1}\right)}\left(d^{k}\right)\right)=\varphi\left(h^{i} x^{j} h^{k \bar{F}} \# d^{k \tau_{1}}\right) \\
& =h^{i} x^{j} h^{k \bar{F}} r_{(f, F)}\left(d^{k \tau_{1}}\right) \# v_{(s, t)}\left(d^{k \tau_{1}}\right)=h^{i} x^{j} h^{k \bar{F}+k \tau_{1} F} \# d^{k \tau_{1} t} \\
& \stackrel{27}{=} h^{i} x^{j} h^{k \bar{F}+k \tau_{1} F} \# d^{k(\tau t-\eta n t)}=h^{i} x^{j} h^{k \bar{F}+k \tau_{1} F} \# d^{k \underline{\underline{2 t}}}=h^{i} x^{j} h^{k\left(\bar{F}+\tau_{1} F\right)} \# d^{k\left(1-n \tau^{\prime}\right)} \\
& \stackrel{20}{=} h^{i} x^{j} h^{-k \beta m} \# d^{k}=h^{i} x^{j} \# d^{k}
\end{aligned}
$$

for all $i, j \in\{0,1, \cdots, m-1\}$ and $k \in\{0,1, \cdots, n-1\}$. By a rather long but straightforward computation one can see that in fact $\varphi \circ \bar{\varphi}=\operatorname{Id}_{T_{2 n m^{2}}^{\bar{\beta}, \bar{\sigma}}(q)}$ and $\bar{\varphi} \circ \varphi=\operatorname{Id}_{T_{2 n m^{2}}^{\beta, \sigma}(q)}$.

Based on Theorem 2.4, we are now able to count the number of types of Hopf algebras which factorize through $T_{m^{2}}(q)$ and $K\left[D_{2 n}\right]$.

Theorem 2.5. Let $m, n \in \mathbb{N}^{*}, m \geq 2, n \geq 3$ and consider $\sharp_{n, m}^{q}$ to be the number of types of Hopf algebras which factorize through $T_{m^{2}}(q)$ and $K\left[D_{2 n}\right]$. Then, we have:

$$
\sharp_{n, m}^{q}= \begin{cases}2, & \text { if } m \text { and } n \text { are both odd } \\ 3, & \text { if } m \text { is odd and } n \text { is even } \\ 1, & \text { if } m \text { and } n \text { are both even or } m \text { is even and } n \text { is odd. }\end{cases}
$$

Proof. According to Theorem 2.2. any Hopf algebra which factorizes through $T_{m^{2}}(q)$ and $K\left[D_{2 n}\right]$ is isomorphic to $T_{2 n m^{2}}^{\beta, \sigma}(q)$ for some $\beta \in U_{2}(K)$ and $\sigma \in U_{(n, n-2)}(K)$.

Suppose first that $n$ is odd. Then $\sigma \in U_{(n, n-2)}(K)$ implies $\sigma=1$ and we are left to decide if the Hopf algebras $T_{2 n m^{2}}^{1,1}(q)$ and $T_{2 n m^{2}}^{-1,1}(q)$ are isomorphic. By Theorem 2.4 we have an isomorphism between the aforementioned Hopf algebras if and only if there exist integers $f, F \in\{0,1, \cdots, m-1\}, s$, $t \in\{0,1, \cdots, n-1\}$ such that $(t, n)=1$ and the following compatibilities hold:

$$
m|2 f, \quad m| n F, \quad m \mid 2 F, \quad q^{f}=-1, \quad q^{F}=1 .
$$

As $q$ is a primitive $m$-th root of unity and $q^{F}=1$, where $F \in\{0,1, \cdots, m-1\}$, we obtain $F=0$. If $m$ is odd as well and $m \mid 2 f$ it follows that $m \mid f$. Now since $f \in\{0,1, \cdots, m-1\}$ we get $f=0$ which contradicts $q^{f}=-1$. Consequently, if $n$ and $m$ are both odd then $T_{2 n m^{2}}^{1,1}(q)$ is not isomorphic to $T_{2 n m^{2}}^{-1,1}(q)$. Hence and we have two types of Hopf algebras which factorize through $T_{m^{2}}(q)$ and $K\left[D_{2 n}\right]$. If $m$ is even and $m=2 m^{\prime}$ for some $m^{\prime} \in \mathbb{N}^{*}$, it is straightforward to see that the integers $s=t=1$, $f=m^{\prime}$ and $F=0$ fulfill the conditions in (30). Therefore, in this case $T_{2 n m^{2}}^{1,1}(q)$ is isomorphic to $T_{2 n m^{2}}^{-1,1}(q)$ and we obtain $\sharp_{n, m}^{q}=1$.

Assume now that $n$ is even and $n=2 \bar{n}$ for some $\bar{n} \in \mathbb{N}^{*}$. Then $\beta, \sigma \in\{1,-1\}$ and the Hopf algebras which factorize through $T_{m^{2}}(q)$ and $K\left[D_{2 n}\right]$ are the following:

$$
T_{2 n m^{2}}^{1,1}(q), \quad T_{2 n m^{2}}^{-1,-1}(q), \quad T_{2 n m^{2}}^{1,-1}(q), \quad T_{2 n m^{2}}^{-1,1}(q) .
$$

If $m$ is odd, $m=2 \bar{m}+1$ for some $\bar{m} \in \mathbb{N}^{*}$, we will see that $T_{2 n m^{2}}^{-1,-1}(q)$ is isomorphic to $T_{2 n m^{2}}^{1,-1}(q)$ while any two of the remaining three Hopf algebras are non-isomorphic. Indeed, it can be readily seen that the integers $s=t=1, f=F=0$ fulfill the compatibility conditions (23) and (24) in Theorem 2.4 which leads us to the conclusion that $T_{2 n m^{2}}^{-1,-1}(q)$ is isomorphic to $T_{2 n m^{2}}^{1,-1}(q)$. Similarly, by 
Theorem 2.4, the Hopf algebras $T_{2 n m^{2}}^{1,1}(q)$ and $T_{2 n m^{2}}^{-1,-1}(q)$ are isomorphic if and only if there exist integers $f, F \in\{0,1, \cdots, m-1\}, s, t \in\{0,1, \cdots, n-1\}$ such that $(t, n)=1$ and the following hold:

$$
m|2 f, \quad m| n F, \quad m \mid 2 F, \quad q^{f}=1, \quad q^{F}=(-1)^{t} .
$$

To start with, notice that $n$ even and $(t, n)=1$ implies $t$ odd and therefore $q^{F}=-1$. Moreover, as $m \mid 2 F$ and $m=2 \bar{m}+1$ we obtain $m \mid F$. Now since $F \in\{0,1, \cdots, m-1\}$ we get $F=0$. However, this contradicts $q^{F}=-1$ and therefore $T_{2 n m^{2}}^{1,1}(q)$ is not isomorphic to $T_{2 n m^{2}}^{-1,-1}(q)$. In the same manner it can be proved that $T_{2 n m^{2}}^{1,1}(q)$ is not isomorphic to $T_{2 n m^{2}}^{-1,1}(q)$ and respectively that $T_{2 n m^{2}}^{-1,-1}(q)$ is not isomorphic to $T_{2 n m^{2}}^{-1,1}(q)$. Hence, in this case we have $\sharp_{n, m}^{q}=3$.

Finally, if $m$ is even one can easily conclude by pursuing a similar strategy that all Hopf algebras $T_{2 n m^{2}}^{\beta, \sigma}(q)$ are in fact isomorphic to $T_{2 n m^{2}}^{1,1}(q)$ and therefore $\sharp_{n, m}^{q}=1$.

\section{REFERENCES}

[1] Agore, A.L. - Hopf algebras which factorize through the Taft algebra $T_{m^{2}}(q)$ and the group Hopf algebra $K\left[C_{n}\right]$, preprint 2016, arXiv: 1611.05674.

[2] Agore, A.L. - Classifying bicrossed products of two Taft algebras, J. Pure Appl. Algebra 222 (2018), 914-930.

[3] Agore, A.L., Bontea, C.G., Militaru, G. - Classifying bicrossed products of Hopf algebras, Algebr. Represent. Theory 17 (2014), 227-264.

[4] Agore, A.L., Chirvăsitu, A., Ion, B., Militaru, G. - Bicrossed products for finite groups. Algebr. Represent. Theory, 12 (2009), 481-488.

[5] Andrada, A., Salamon, S. - Complex product structures on Lie algebras, Forum Math., 17 (2005), 261-295.

[6] Angiono, I., Galindo, C., Vendramin, L. - Hopf braces and Yang-Baxter operators, Proc. AMS 145 (2017), $1981-1995$.

[7] Bontea, C.G. - Classifying bicrossed products of two Sweedler's Hopf algebras, Czech. Math. J. 64 (2014), $419-431$.

[8] Caenepeel, S., Ion, B., Militaru, G., Zhu, S. - The factorization problem and the smash biproduct of algebras and coalgebras. Algebr. Represent. Theory 3 (2000), 19-42.

[9] Cap, A., Schichl, H., Vanzura, J. - On twisted tensor product of algebras, Comm. Algebra 23 (1995), 4701 - 4735.

[10] Gateva-Ivanova, T., Majid, S. - Matched pairs approach to set theoretic solutions of the Yang-Baxter equation, J. Algebra 319 (2008), 1462-1529.

[11] Golasiński, M., Gonçalves D. - On automorphisms of split metacyclic groups, Manuscripta Math. 128 (2009), $251-273$.

[12] Guccione, J.A., Guccione, J.J., Valqui, C. - Twisted planes, Comm. Algebra 38 (2010), 1930-1956.

[13] Kassel, C. - Quantum groups, Graduate Texts in Mathematics 155. Springer-Verlag, New York, 1995.

[14] Keilberg, M. - Automorphisms of the doubles of purely non-abelian finite groups, Algebr. Represent. Theory 18 (2015), 1267-1297.

[15] Kosmann-Schwarzbach, Y., Magri, F. - Poisson-Lie groups and complete integrability. I. Drinfel'd bialgebras, dual extensions and their canonical representations, Ann. Inst. H. Poincar Phys. Thor. 49 (1988), 433-460.

[16] Majid, S. - Matched pairs of Lie groups and Hopf algebra bicrossproducts, Nuclear Physics B 6 (1989), $422-424$.

[17] Majid, S. - Physics for algebraists: non-commutative and non-cocommutative Hopf algebras by a bicrossproduct construction, J. Algebra 130 (1990), 17-64.

[18] Jara, P., López Peña, J., Navarro, G., Ştefan, D. - On the classification of twisting maps between $K^{n}$ and $K^{m}$, Algebr. Represent. Theory 14 (2011), 869-895.

[19] Takeuchi, M. - Matched pairs of groups and bismash products of Hopf algebras, Comm. Algebra 9 (1981), 841-882.

Faculty of Engineering, Vrije Universiteit Brussel, Pleinlaan 2, B-1050 Brussels, Belgium and "Simion Stoilow" Institute of Mathematics of the Romanian Academy, P.O. Box 1-764, 014700 Bucharest, Romania

E-mail address: ana.agore@vub.be and ana.agore@gmail.com

'Simion Stoilow" Institute of Mathematics of the Romanian Academy, P.O. Box 1-764, 014700 Bucharest, Romania

E-mail address: lauranastasescu@gmail.com 